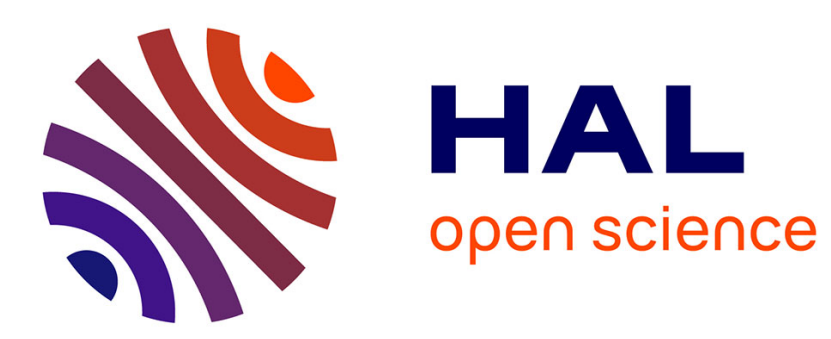

\title{
A new approach for the simultaneous tracking of multiple honeybees for analysis of hive behavior
}

Toshifumi Kimura, Mizue Ohashi, Ryuichi Okada, Hidetoshi Ikeno

\section{To cite this version:}

Toshifumi Kimura, Mizue Ohashi, Ryuichi Okada, Hidetoshi Ikeno. A new approach for the simultaneous tracking of multiple honeybees for analysis of hive behavior. Apidologie, 2011, 42 (5), pp.607-617. 10.1007/s13592-011-0060-6 . hal-01003596

\section{HAL Id: hal-01003596 https://hal.science/hal-01003596}

Submitted on 1 Jan 2011

HAL is a multi-disciplinary open access archive for the deposit and dissemination of scientific research documents, whether they are published or not. The documents may come from teaching and research institutions in France or abroad, or from public or private research centers.
L'archive ouverte pluridisciplinaire HAL, est destinée au dépôt et à la diffusion de documents scientifiques de niveau recherche, publiés ou non, émanant des établissements d'enseignement et de recherche français ou étrangers, des laboratoires publics ou privés. 


\title{
A new approach for the simultaneous tracking of multiple honeybees for analysis of hive behavior
}

\author{
Toshifumi Kimura $^{1}$, Mizue Ohashi ${ }^{1}$, Ryuichi OKadA $^{2}$ and Hidetoshi IKeno $^{1}$ \\ ${ }^{1}$ School of Human Science and Environment, University of Hyogo, 1-1-12, Shinzaike-honcho, Himeji, Hyogo \\ 670-0092, Japan \\ ${ }^{2}$ Kagawa School of Pharmaceutical Sciences, Tokushima Bunri University, 1314-1, Shido, Sanuki, Kagawa \\ 769-2193, Japan
}

Received 1 July 2010 - Revised 14 December 2010 - Accepted 3 January 2011

\begin{abstract}
Social activities are among the most striking of animal behaviors, and the clarification of their mechanisms is a major subject in ethology. Honeybees are a good model for revealing these mechanisms because they display various social behaviors, such as division of labor, in their colonies. Image processing is a precise and convenient tool for obtaining animal behavior data, but even recent methods are inadequate for the identification or description of honeybee behavior. This is because of the difficulty distinguishing between the large number of individuals in a small hive and their multiple movements. The present study developed a new computer-aided system, using a vector quantization method, for the identification and behavioral tracking of individual honeybees. The vector quantization method enabled separation of honeybee bodies in photographs recorded as a movie. This system succeeded in analyzing a huge number of frames quickly and can thus save both time and labor. Moreover, the system identified more than $72 \%$ of the bees in a hive and found and determined the active areas in the hive by extracting the trajectories of walking bees. In addition, useful behavioral data on the honeybee waggle dance were obtained using the present system.
\end{abstract}

\section{behavioral tracking / honeybee / Apis mellifera / image processing}

\section{INTRODUCTION}

Social activities are among the most striking of animal behaviors, and the clarification of their mechanisms is a major subject in ethology (Manning and Dawkins 1998). Honeybees are a good model for revealing these mechanisms because they display various social behaviors such as division of labor (Gould and Gould 1988; Tautz and Hubland 2008). A honeybee colony consists of tens of thousands of worker bees, one queen, and some hundreds of drones. While the queen expends her energy laying eggs, worker bees, depending on their age,

Corresponding author: T. Kimura,

kimura@shse.u-hyogo.ac.jp

Manuscript editor: Monique Gauthier clean and build the hive, take care of the brood and the queen, defend the hive from enemies, and leave the nest to search around the hive and collect food (Frisch 1967; Gould and Gould 1988; Tautz and Hubland 2008). Thus, individual bees exhibit different behaviors within the hive.

A unique communication behavior in honeybees, to share information regarding profitable food sources, was discovered by von Frisch (Frisch 1967). After a forager bee has returned to the hive from a successful foraging flight, it often performs a waggle dance to transfer information regarding the direction and distance of the discovered food source to other worker bees. Some workers (followers) surround the dancing bee (dancer), receive the information and then visit the food source that the dancer 
has indicated (Dyer 2002; Esch et al. 2001; Frisch 1967; Gould and Gould 1988; Seeley 1995; Seeley and Visscher 1988).

To understand the mechanisms involved in such social behaviors, we must be able to precisely identify individual bees and provide a detailed description of their behaviors. These tasks have generally been achieved by manual analysis of film or video footage. For example, thousands of bees would be marked for an observation of a hive by means of numbers and/ or color combinations (Abramson and Bozic 2004; Gould and Gould 1988; Kirchner and Lindauer 1998; Seeley 1995; Seeley and Visscher 1988; Tautz 1996; Visscher and Seeley 1982). Some of the dancers and followers might then be tracked (Seeley 1994; Judd 1995) and various dance positions plotted (Seeley and Towne 1992; Tautz and Lindauer 1997). Thus, the dance precision was calculated in many behavioral experiments (Beekman et al. 2005; De Marco et al. 2008; Okada et al. 2008a, b; Towne and Gould 1988; Weidenmuller and Seeley 1999). However, such manual analyses require large amounts of time and labor.

Image processing is a useful method for the identification and tracking of multiple moving elements (Bodor et al. 2003; Bose et al. 2007; Zhao and Tao 2005). Some methods have been developed for studying sequential photographs of these movements, for example, tracking ants by body color and movement information (Balch et al. 2001); automatic identification of bees using a combination of k-nearest neighbor classification and a hidden Markov model (Feldman and Balch 2003); and tracking of unmarked bees using a Rao-Blackwellized particle filter and several principal images of bees (eigen-bees) (Khan et al. 2003). However, it is difficult to directly apply these methods for analyzing the social behavior of the animal group because they are developed for processing independent behaviors of a few individuals. One problem is that a background image with no tracking object is required for the extraction of tracking objects (Balch et al. 2001; Bodor et al. 2003; Bose et al. 2007; Feldman and Balch 2003; Khan et al. 2003; Zhao and Tao 2005).
Another is that marks on and/or numbers of tracking objects to be analyzed may also be required (Balch et al. 2001; Bodor et al. 2003; Khan et al. 2003). Methods developed for tracking a few individuals are generally inadequate for simultaneously tracking large numbers of individuals (Bodor et al. 2003; Bose et al. 2007; Feldman and Balch 2003). There are no methods that can simultaneously track hundreds of unmarked targets in a changing background. Thus, the development of suitable methods for analyzing animal behaviors is still worthy of considerable attention.

In the present study, a computer-aided system was developed for the identification and behavioral tracking of individual honeybees within a hive. The system was based on vector quantization and temporal contextual information. The vector quantization method (VQ) allowed honeybee bodies in time series photographs to be separated from one another without any preconditions. Individual bees were extracted from images using their body size and locomotion. This new system successfully tracked hundreds of bees simultaneously without the need for marking. The system identified more than $72 \%$ of the bees in the hive and successfully traced the trajectory of the waggle dance.

\section{METHODS}

\subsection{Procedure}

Despite widespread interest in the social behavior of honeybees, a number of factors, such as their small body size, the large population within a hive and the lack of pronounced external features on individual bees, make behavioral analyses problematic. To overcome these problems the following three steps were taken in the development of the analysis system: (a) extraction of a honeybee-code image from the whole hive image using the VQ; (b) separation of single and plural regions of honeybees from the honeybee-code image using an average honeybee body size and shape; (c) tracking of honeybee movement from temporal contextual information in sequential image data or movies. 


\subsection{Extraction of honeybee body regions using VQ}

VQ is used to separate many elements into several groups with approximately the same characteristics. Each group is represented by a centroid vector, which is called the "code vector" (CV) (Chen et al. 2000). This method has been applied to various fields, such as data compression, signal processing, and data clustering.

Normally, a color image consists of three color components: red (R), green (G), and blue (B). From quantization of the $R, G$, and $B$ components of images, $\mathrm{R}$ was found to be the best. It seems likely that this reflects the warm colors such as yellow and orange which predominate in the honeybee body. Thus, the highest contrast image was obtained from the $\mathrm{R}$ image (Figure 1a). This image was split into small regions of $2 \times 2$ pixels, and each region was categorized according to spread and the sharpness of the image. Based on the 256 levels of each pixel each $2 \times 2$ pixel region mapped a point (training vector (TV)) in four-dimensional space. Analyses were investigated using regions of different pixel numbers $(3 \times 3,4 \times 4$, and more). Increasing the pixel number within each region increases the space mapped but also increases distance between TVs and thus reduces the resolution of the image. The $2 \times 2$ pixel regions were found to be the best for the separation of the honeybee individuals from their background.

In VQ applications the generation of good CV values is critical. Previously, it was extremely difficult to calculate $\mathrm{CV}$ values from the image data. However, the Linde-Buzo-Gray algorithm (Gray 1984; Linde et al. 1980) used in the current study automatically generated CV values from TV values using the following iterative process.

1. An initial $\mathrm{CV}$ (e.g., $\mathrm{CV}_{0}$ ) value was calculated using the average of all $\mathrm{TV}$ values.

2. A first $\mathrm{CV}$ (e.g., $\mathrm{CV}_{1}$ and $\mathrm{CV}_{2}$ ) value was generated from the initial $\mathrm{CV}\left(\mathrm{CV}_{0}\right)$ value by the addition and subtraction of a small value to and from the initial $\mathrm{CV}$ value, respectively.

3. The TV values were separated into two groups $\left(\mathrm{TV}_{1}\right.$ and $\mathrm{TV}_{2}$ ) based upon the new $\mathrm{CV}$ values $\left(\mathrm{CV}_{1}\right.$ and $\left.\mathrm{CV}_{2}\right)$.

4. The two $\mathrm{CV}$ values $\left(\mathrm{CV}_{1}\right.$ and $\left.\mathrm{CV}_{2}\right)$ were then each recalculated using the average of the relevant reclassified TV values (i.e., from each $\mathrm{TV}_{1}$ and $\mathrm{TV}_{2}$ ).

5. The process was repeated, adding further $\mathrm{CV}$ values and TV groups until a minimum Euclidean distance between the $\mathrm{CV}$ values and TV values was reached.

6. Each CV was split into two vectors again. The same operation was carried out in a target vector space.

In a preliminary experiment, the number of $\mathrm{CV}$ values for classification was varied. It was found that eight $\mathrm{CV}$ values produced the best result for the separation of objects within the observation hive. These vectors correspond to the honeybee body, the dark and light of the honeybee wing, the hive, the hive frame, and the background and noise. Thus, eight CVs were needed to represent all of the objects. The vector distribution and quantization results for each region are shown in Figure 2. Each vector consists of four dimensions $(v 1, v 2, v 3, v 4)$. The distribution of $\mathrm{CV}$ values was examined in 2 twodimensional graphs $(v 1-v 2$ plane, Figure $2 \mathrm{a}$ and $v 3-$ $v 4$, Figure 2b). The CV values, cv3 and cv5 representing the hive and honeybee body regions, respectively, are approximately at the center of those regions. In most cases, TV values could classify the central CV values for each region. The system employed in this study generated eight $\mathrm{CV}$ values from all of the TV values and extracted an image for honeybee body regions, called a "honeybee-code image," from each frame.

\subsection{Separation of a honeybee-code image into single honeybee regions and plural honeybee regions}

Two different types of regions in the honeybeecode image were extracted from the hive image. The first type was a single honeybee region (SHR) which consisted of a single honeybee, and the second was a plural honeybee region (PHR) which consisted of a group of two or more honeybees. First all of the SHRs were extracted from the hive image based upon the morphological characteristics of honeybees (Figure 1c). Subsequently, the PHRs were processed to separate individuals (Figure 1d). Morphological information, including the body size and shape of 

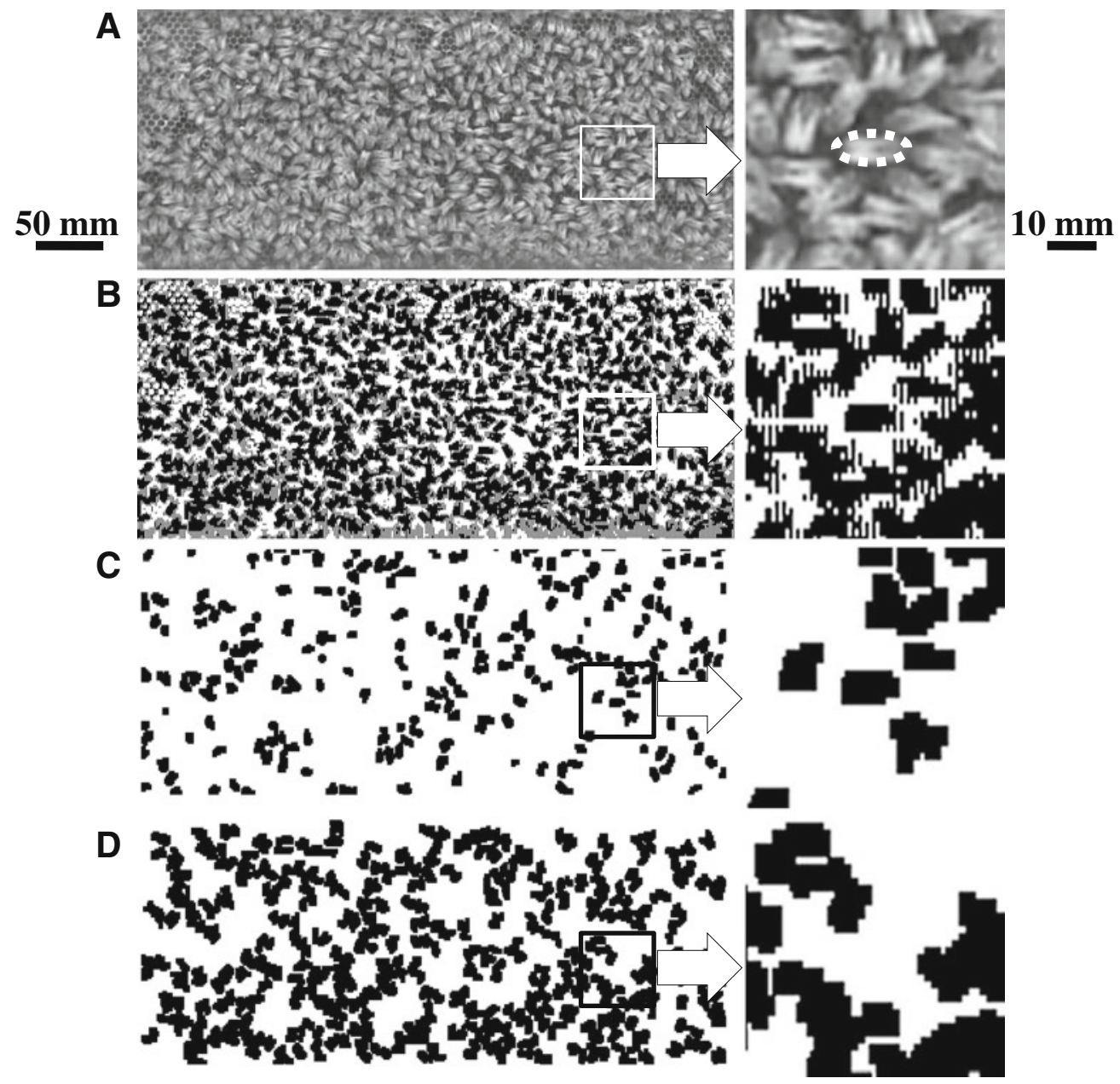

Figure 1. The honeybee-code image and honeybee region. a A whole hive image. b A honeybee-code image obtained using VQ and the whole hive image a. The detected honeybee regions are indicated in black in b. c, d After repeating our procedure, a single honeybee region image $\mathbf{c}$ was obtained, which consisted of single honeybee regions alone. By subtraction of the single honeybee region image from the honeybee-code image, the remaining image was defined as a plural honeybee region image $\mathbf{d}$. The boxed areas to the right of each main image a-d are enlarged for comparison with the original images. The dotted oval in the enlarged area of $\mathbf{a}$ shows the approximation of the honeybee body to an ellipse shape.

single honeybees from the images, was used for detection of the SHR. The shape of a honeybee was approximated visually as an ellipse in Figure 1a, and the size of the SHR estimated as $100 \pm 18$ pixels from 20 manually selected ellipses. Similar sized regions (from 80 to 120 pixels) were extracted as the other SHRs from Figure $1 \mathrm{~b}$.

After the SHRs were processed, the individual honeybees in these regions were identified. Over- lapping areas between the honeybee regions were noted. There were two possible overlapping areas in the SHR. The first type consisted of a temporal overlap between two SHRs that were in fact the same bee (Figure 3a). That is where the SHR for a bee \#1 at time $t$ is $S^{1}{ }_{t}$ and at time $t+1$ the SHR is $S^{1}{ }_{t+1}$ and the regions $S^{1}{ }_{t}$ and $S^{1}{ }_{t+1}$ overlap. The two images needed to be superimposed to identify the two honeybees as identical. 
Figure 2. The vector distribution and quantization results for each region. The position of each CV is shown as a box and is labeled. Other symbols represent vectors generated using VQ. The solid lines show the borders of each region. Four dimensions were used to generate these twodimensional graphs. In most cases, the training vectors could determine the central $\mathrm{CVs}$ for each region.
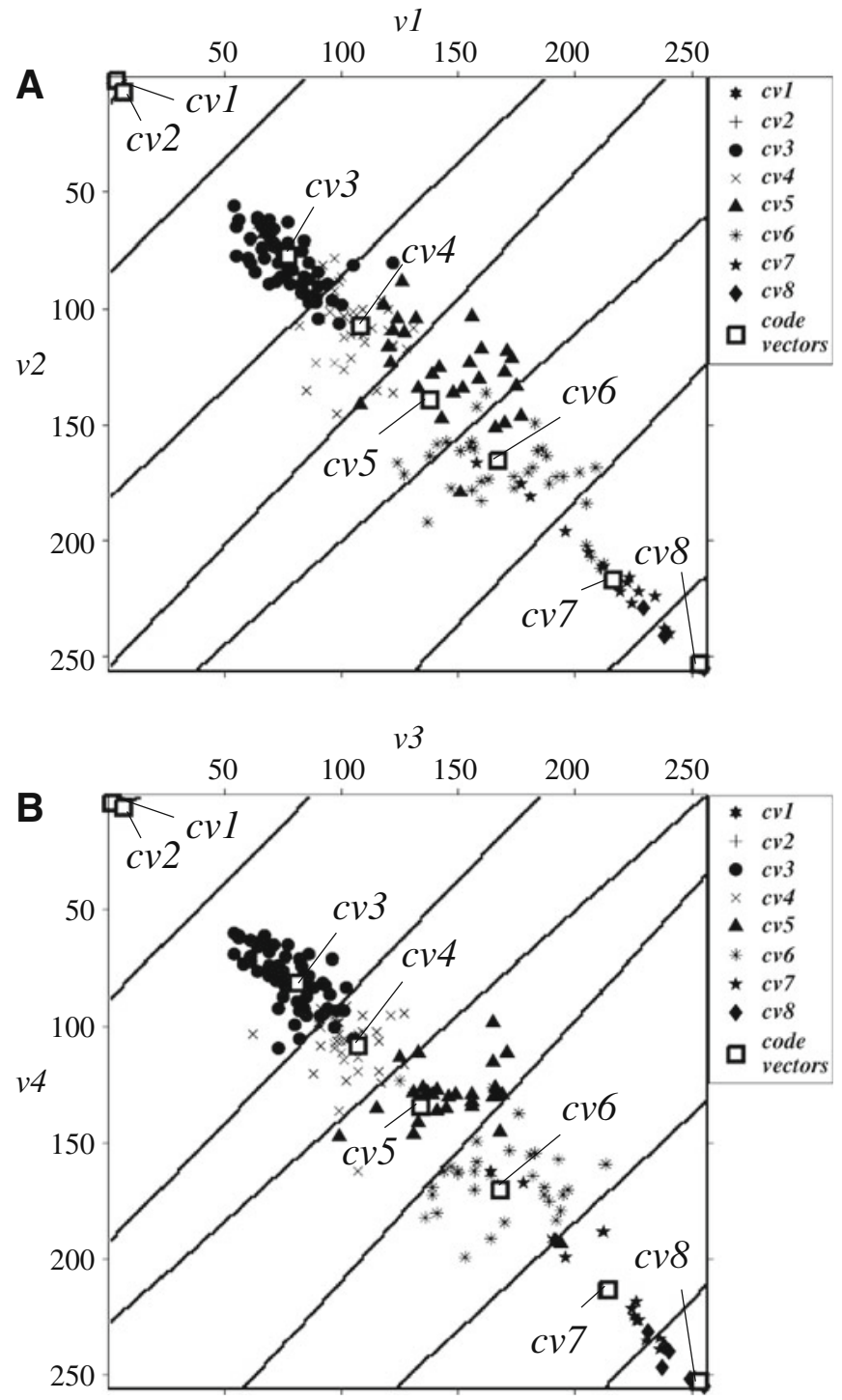

The second type of SHR consisted of an overlap among two or more SHRs (Figure 3b). When SHR $\left(S_{t+1}\right)$ overlapped with two or more SHRs at time $t$, the sizes of the overlapping regions were compared. For example, the size of the overlap between $S_{t+1}$ and $S^{2}{ }_{t}$ were compared that between $S_{t+1}$ and $S^{3}{ }_{t}$. It was assumed that the largest overlap occurred between the same bee at different times. That is, the overlapping area between $S_{t+1}$ and $S^{2}$ was larger than that between $S_{t+1}$ and $S^{3}{ }_{t}$ thus $S_{t+1}$ and $S^{2}{ }_{t}$ represent the same bee and $S_{t+1}$ can be renamed $S_{t+1}^{2}$.
When PHRs were divided into SHRs using before and after frames, the identification of the honeybees was more complicated. When a PHR was separated into two or more SHRs, the honeybees were identified as follows (Figure 3c). A PHR at time $t$ was named $P_{t}$. If this PHR overlaps with two SHRs at time $t-1$, i.e., $S_{t-1}^{4}$ and $S_{t-1}^{5}$ then it is likely that the PHR $P_{t}$ actually consisted of two SHRs $S_{t}{ }_{t}$ and $S_{t}^{5}$. Thus, the PHR is separated and identified and from then on is processed as an SHR. This process was repeated until no more PHRs existed which could be processed. 


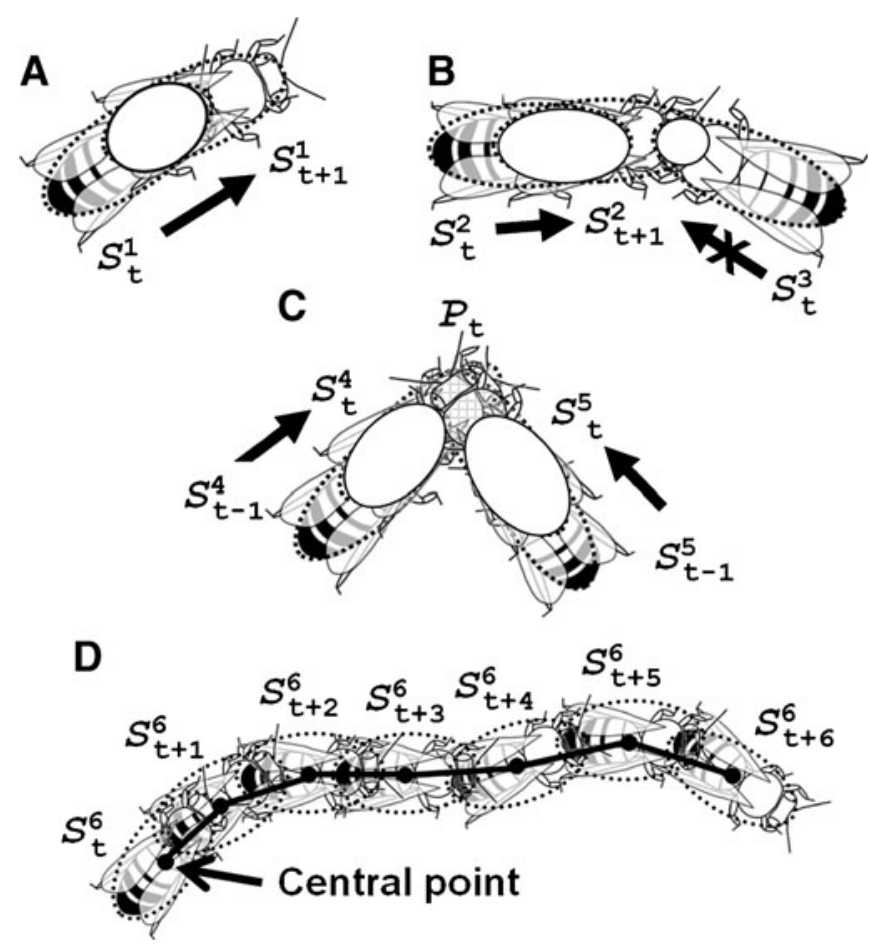

Figure 3. Numbering and tracking of individual honeybees. Open circles show the temporary areas of overlap between bees or bee images. The dotted ovals around the bees show the approximation of the honeybee body as an ellipse. a When a single honeybee region $S_{t+1}$ at a given time $t+1$ was overlapped with one in the preceding image $\left(S_{t}^{1}\right)$, we assigned the same individual number to this region, i.e., bee number \#1 in this case. $\mathbf{b}$ When a single honeybee region was temporally overlapped with more than one single honeybee region, the individual number belonging to the largest overlapped region was assigned, i.e., bee number $\# 2$ in this case. $\mathbf{c}$ When two honeybee regions were merged into one region, the region was separated into two single honeybee regions by examining movement in previous and subsequent frames in this region. $\mathbf{d}$ The trajectory of a honeybee. The central points of an individual that was assigned the same number were connected along a temporal sequence.

Individual movements were tracked by calculating central points by averaging the coordinates of each extracted area. Central points calculated for the same honeybee for different times were connected sequentially. The honeybee trajectory was thus extracted (Figure 2d).

\section{EXPERIMENTS AND RESULTS}

\subsection{Experimental setup}

The behavior of honeybees, Apis mellifera, in an observation hive was recorded as a movie using a digital video camera (GR-HD1; JVC, Yokohama, Japan). The resolution was $720 \times$
480 pixels; the video frame rate was 29.97 frames per second, and the movie was recorded for a bee hive frame (width, $44.0 \mathrm{~cm} \times$ height, $19.6 \mathrm{~cm}$ ) (Figure 4a). In the movie, there were more than 700 honeybees. They had not been marked and could walk freely in the hive.

\subsection{Identification of honeybees}

A 10-s movie (300 frames) was processed. The system was able to automatically number more than 500 honeybees in each frame (Figure 4b). Three frames were selected at random, and we calculated the percentage of correct identification by our software to the total 

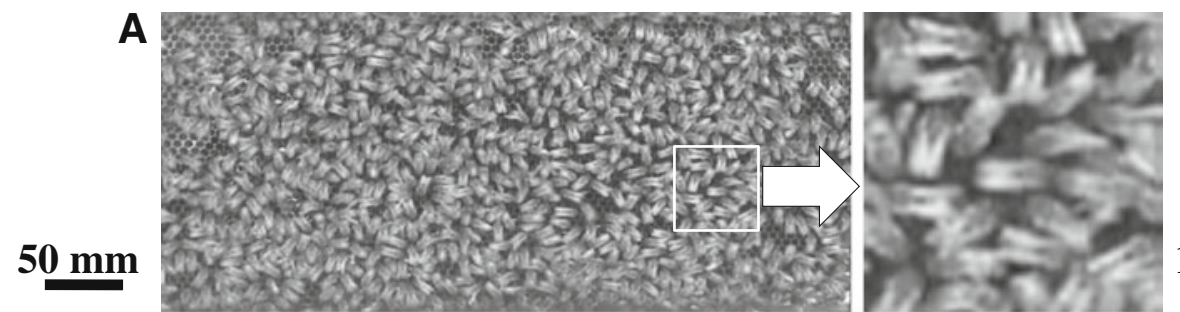

\section{$10 \mathrm{~mm}$}

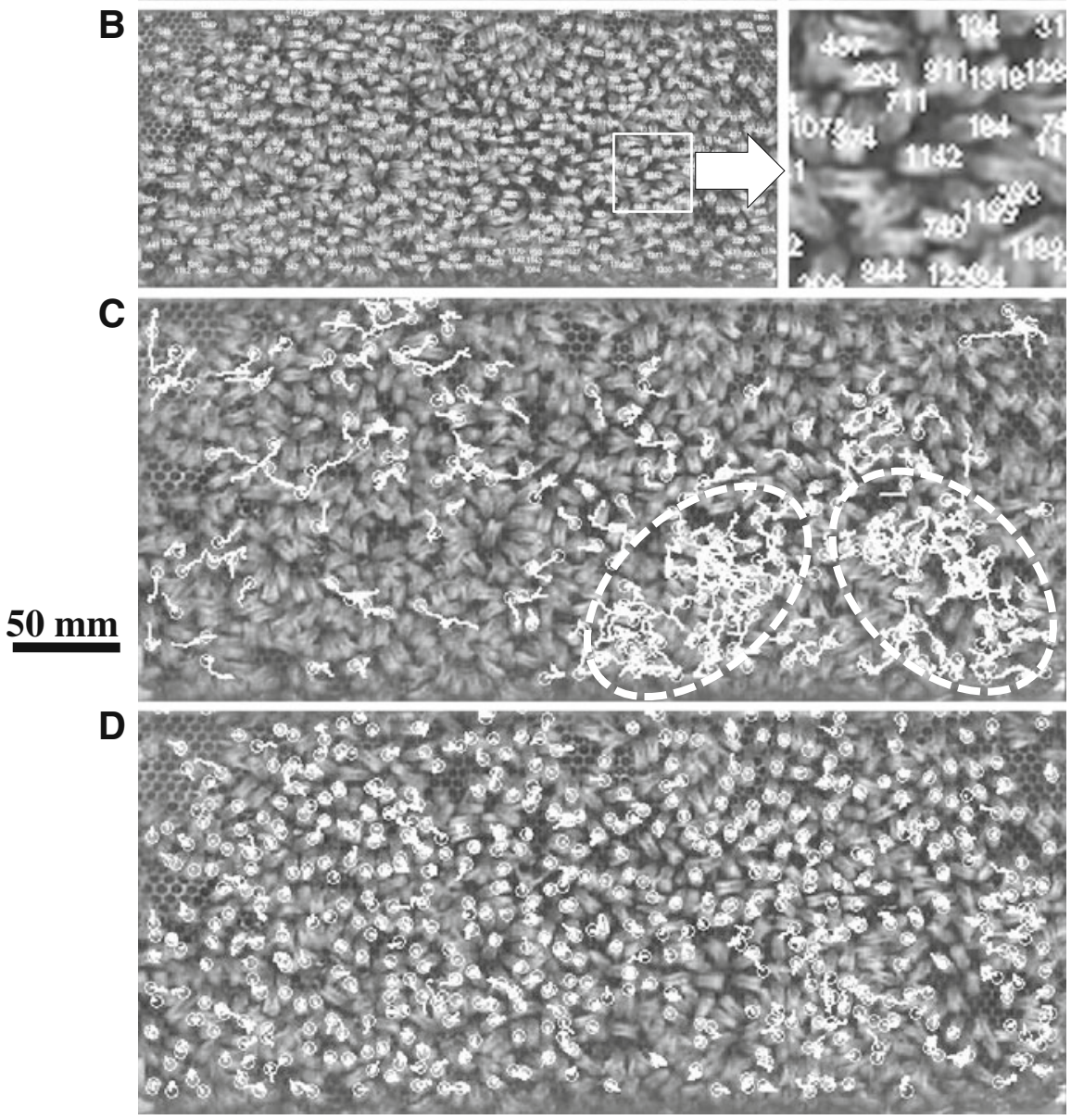

Figure 4. The identification and trajectories of bees. a A whole hive image. Only the red component of a color movie was used to create the hive image. b Identification of honeybees. The number on each honeybee indicates the individual number assigned using the study method. Enlarged images for $\mathbf{a}$ and $\mathbf{b}$ are shown on the right hand side. Most bees were identified, and the system only failed to detect a small number. $\mathbf{c}, \mathbf{d}$ Trajectories of bees walking a long distance (c) or short distance (d). Open circles superimposed on the relevant individuals indicate starting positions. The method identified two areas of high activity in $\mathbf{c}$. These are indicated using a dotted line on the image. The area to the bottom right was particularly active; this area was near the entrance of the hive and is well-known as the site of the waggle dances performed by bees returning from foraging. Indeed, dancing behavior was found in this area at this time. 
number of individuals in each frame. The total number was a number of honeybees extracted and identified manually. More than $72 \%$ of the bees manually identified were also identified using the analysis system (510 of 704, 522 of 718,516 of 700). This result indicates that most bees in a hive can be identified. The only limitation was that bees which only walk only a short distance overlap with other bees and these individuals were difficult to identify.

\subsection{Tracking of honeybee movement}

Individual trajectories were plotted from sequential temporal identification of individuals. The system succeeded in simultaneously tracking hundreds of honeybees in a single hive, resulting in the production of trajectories for more than $50 \%$ of all of the bees. As expected from the analysis of overlapping areas between single and plural honeybee regions, the tracking was easy for honeybees displaying characteristic movements, such as long distance walks and the waggle dance, but the tracking was difficult for bees that did not walk over a long distance. Thus, long distance walkers (Figure 4c) and short distance walkers (Figure 4d) were defined according to whether or not a honeybee moved more than $30 \mathrm{~mm}$ in $10 \mathrm{~s}$.

The trajectories of long distance walkers determined in this study revealed the presence of active areas and static areas within the hive. Figure $4 \mathrm{c}$ shows the location of two active areas in the lower right part of the hive frame. As these areas were near the entrance of the hive, they corresponded to the site of dances performed by workers returned from foraging (Seeley 1994; Seeley and Towne 1992).

\subsection{Extraction of waggle dance}

In a hive, some bees have specific behaviors. The system employed in this study succeeded in extracting the trajectories of some of these, such as the waggle dance. The waggle dance consists of a combination of straight waggling walking and right- or left-turn walking to generate a "dance" in a figure eight. The white lines of
Figure 5 show an example of the waggle dance in one of the active areas extracted using the system. Several parameters, such as the timing of, the duration (coding the distance of the food source from the hive), and the direction (indicating the direction of the food source from the hive) of the straight waggling walk can be calculated from this extraction. These data could be very valuable for ethological studies of honeybees. In Figure 5, the honeybee performed two waggle walks within $10 \mathrm{~s}$. The analysis system developed and employed in this study obtained the behavioral parameters of the waggle dance as obtained by manual tracking. From the system, the durations of these walks were 1.10 and $0.80 \mathrm{~s}(1.10,0.87 \mathrm{~s}$ from manual). The directions were $138.3^{\circ}$ and $143.1^{\circ}\left(131.6^{\circ}\right.$ and $145.0^{\circ}$ in manual).

\section{DISCUSSION}

A new tracking system for honeybee behavior was developed utilizing a VQ. This system had two distinct advantages for identification and tracking. First, the system successfully identified about 500 of more of the 700 bees crowded into a small hive, including overlapping bees. This number is substantially greater than numbers extracted in previous studies where only several tens of the target insects were separated in the image (Khan et al. 2003). Second, this system simultaneously tracked about 350 bees, $50 \%$ of the entire hive. This is much more than the number of individuals tracked in previous studies, e.g., one bee (Khan et al. 2004), seven ants (Balch et al. 2001), or 20 ants (Khan et al. 2003). Nonetheless, although this new method can track the behavior of most honeybees either extracted as single or plural regions, there are some circumstances where this method does not work. Such cases are when one bee stands directly on top of another bee or when bees hide in holes in the hive structure. Thus, there are additional improvements that can be made for tracking such complex behaviors.

Further evidence of the power of this new system was its ability to track hundreds of 

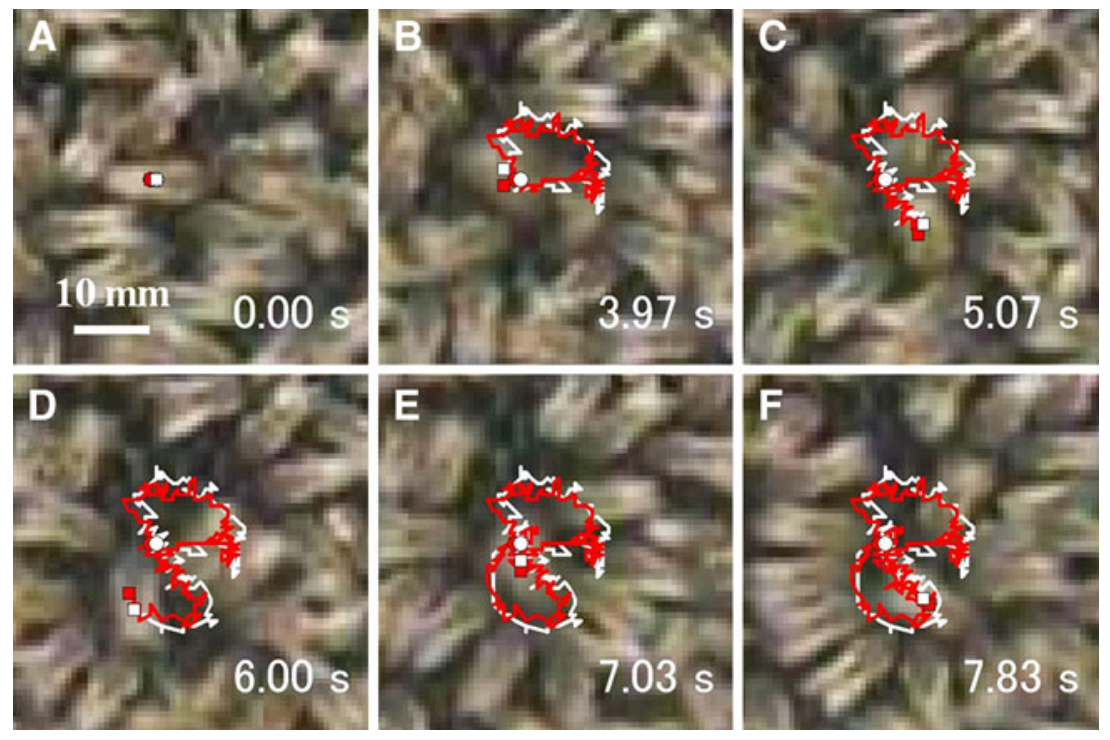

Figure 5. The trajectory of a dancer during a waggle dance. The white and red lines indicate the trajectories described by automatic and manual tracking methods, respectively. The dancer indicated by the open circle (a) was tracked during one cycle of a waggle dance. The number in the lower right of each image shows the time elapsed from the start of tracking. First, the bee moved in a wide circle from and back to the initial position (b). Then the bee made a first waggle walk (c). Next, the bee turned right and walked in a straight line (d), then returned to her initial position (e). Lastly, the bee made a second waggle walk (f). Overall, the path taken follows a figure eight.

objects under the relatively poor conditions. For example, the resolution of the images was $720 \times$ 480 pixels for $44 \times 20 \mathrm{~cm}$, which is close to those used by Balch et al. (Balch et al. 2001) $(640 \times 480$ pixels, $40 \times 20 \mathrm{~cm})$, but lower than that used by Khan et al. (Khan et al. 2003) $(720 \times 480$ pixels, $15 \times 10 \mathrm{~cm})$. Our analyses were performed using natural sunlight, which is much poorer and less controlled than the light sources generally used in these experiments. Furthermore, the complexity caused by dynamic change in images is compounded as the number of targets is increased. Thus, tracking hundreds or thousands of objects also is much more difficult than tracking small numbers.

Our system could process a movie up to $3 \mathrm{~min}$ due to memory limitation. For a longer time movie, it is necessary to split it into the series of processable time movies. The utilization of a computer with a high-capacity memory will solve this problem. We can also process the longer time movie by reducing the frame rate, but it is not recommended because some important information of movements, such as overlapping, might be lost by the deduction, which will decrease the accuracy of tracking.

It is expected that the accuracy of tracking would decrease with time due to the complex movements of individuals. However, this problem will be avoided by modifying the trajectories of bees manually and visually as necessary. Additionally, the following attentions are recommended during image recording: (1) the focus of the digital video camera is accurate on the whole hive during the record, (2) the lighting is uniform on the whole hive, (3) a homogenous background and new combs are used for the record.

This computer-aided system using VQ was found to be highly applicable for bee experiments. A single colony of social insects like honeybees contains a large number of individuals. Conventional computational methods are limited by CPU, memory, and other hardware ceilings and cannot meet the requirements of 
complex processing to deal with data sets for such a large number of targets simultaneously. However, VQ can select the essential information and drop the unessential data, thereby reducing the workload for the computer (Gersho and Gray 1992) and enabling the analysis of hundreds of bees simultaneously. To identify and track 700 individual bees manually would require more than 50 days for 11 frames of a 10$\mathrm{s}$ movie. Using the present system, this required only $1 \mathrm{~h}$ for 300 frames of the same movie.

These results indicate that the developed system is an effective tool for behavioral analyses in honeybee experiments. In addition, the system was able to at least partly resolve the problem of overlapping honeybees. Making improvements in the accuracy of individual identification by using a high resolution digital video camera and a motion estimation algorithm could resolve this problem. This system should ultimately not only be applicable to honeybees but also to the social behavior of human beings.

\section{ACKNOWLEDGMENTS}

This work was partially supported by University of Hyogo, the Sanyo Special Steel Cultural Promotion Foundation, and a KAKENHI Grant (no. 20033023) for Scientific Research on the Priority Area "Emergence of Adaptive Motor Function through Interaction between Body, Brain and Environment" from the Japanese Ministry of Education, Culture, Sports, Science and Technology.

Analyse du comportement dans la ruche: une nouvelle approche pour le suivi simultané de plusieurs abeilles.

suivi comportemental / abeille / Apis mellifera / enregistrement du comportement / traitement de l'image

\section{Eine neue Methode für die gleichzeitige Beobachtung von zahlreichen Bienen zur Verhaltensanalyse in einem Bienenvolk}

Verhaltensbeobachtung / Honigbiene / Apis mellifera / Bildbearbeitung

\section{REFERENCES}

Abramson, C.I., Bozic, J. (2004) Multiple search flights of the dancer's followers: result of information or energy deficiency. Apiacta 38, 358-365

Balch, T., Khan, Z., Veloso, M. (2001) Automatically tracking and analyzing the behavior of live insect colonies. Proceedings of the Fifth International Conference on Autonomous Agents, pp. 521-528

Beekman, M., Doyen, L., Oldroyd, B.P. (2005) Increase in dance imprecision with decreasing foraging distance in the honey bee (Apis mellifera L.) is partly explained by physical constraints. J. Comp. Physiol. A 191, 1107-1113

Bodor, R., Jackson, B., Papanikolopoulos, N. (2003) Vision-based human tracking and activity recognition. Proceedings of the 11th Mediterranean Conference on Control and Automation [CD-ROM], T7-30

Bose, B., Wang, X., Grimson, E. (2007) Multi-class object tracking algorithm that handles fragmentation and grouping. Proceedings of the IEEE Conference on Computer Vision and Pattern Recognition 2007, pp. 1-8

Chen, D., Liang, Z., Wax, M.R., Li, L., Li, B., Kaufman, A.E. (2000) A novel approach to extract colon lumen from CT images for virtual colonoscopy. IEEE Trans. Med. Imaging 19, 1220-1226

De Marco, R.J., Gurevitz, J.M., Menzel, R. (2008) Variability in the encoding of spatial information by dancing bees. J. Exp. Biol. 211, 1635-1644

Dyer, F.C. (2002) The biology of the dance language. Annu. Rev. Entomol. 47, 917-949

Esch, H., Zhang, S., Srinivasan, M., Tautz, J. (2001) Honey bee dances communicate distances measured by optic flow. Nature 411, 581-583

Feldman, A., Balch, T. (2003) Automatic identification of bee movement. Proceedings of the 2nd International Workshop on the Mathematics and Algorithms of Social Insects, pp. 53-59

Frisch, K.V. (1967) The dance language and orientation of bees. Harvard University Press, Cambridge

Gersho, A., Gray, R.M. (1992) Vector quantization and signal compression. Kluwer Academic Publishers, Norwell

Gould, J.M., Gould, C.G. (1988) The honey bee. Scientific American Library, New York

Gray, R.M. (1984) Vector quantization. IEEE ASSP Mag. 1, 4-29

Judd, T.M. (1995) The waggle dance of the honey bee: which bees following a dancer successfully acquire the information? J. Insect Behav. 8, 343-354

Khan, Z., Balch, T., Dellaert, F. (2003) Efficient particle filter-based tracking of multiple interacting targets using an MRF-based motion model. Proceedings of the 2003 IEEE/RSJ International Conference on Intelligent Robots and Systems 1, pp. 254-259

Khan, Z., Balch, T., Dellaert, F. (2004) A Rao-Blackwellized particle filter for EigenTracking. Proceedings of the 
IEEE Computer Society Conference on Computer Vision and Pattern Recognition 2004, pp. 980-986

Kirchner, W.H., Lindauer, M. (1998) Honeybee dance communication, acoustical indication of direction in round dances. Naturwissenschaften 75, 629630

Linde, Y., Buzo, A., Gray, R.M. (1980) An algorithm for vector quantizer design. IEEE Trans. Comm. 28, 84-95

Manning, A., Dawkins, M.S. (1998) An introduction to animal behaviour. Cambridge University Press, Cambridge

Okada, R., Ikeno, H., Sasayama, N., Aonuma, H., Kurabayashi, D., Ito, E. (2008a) The dance of the honeybee: how do honeybees dance to transfer food information effectively? Acta Biol. Hung. 59 (Suppl), 157-162

Okada, R., Ikeno, H., Aonuma, H., Ito, E. (2008b) Biological insights into robotics: honeybee foraging behavior by a waggle dance. Adv Robotics 22, 1665-1681

Seeley, T.D. (1994) Honey bee foragers as sensory units of their colonies. Behav. Ecol. Sociobiol. 34, 51-62

Seeley, T.D. (1995) The wisdom of the hive: the social physiology of honey bee colonies. Harvard University Press, Cambridge

Seeley, T.D., Towne, W.F. (1992) Tactics of dance choice in honey bees: do foragers compare dances? Behav. Ecol. Sociobiol. 30, 59-69
Seeley, T.D., Visscher, P.K. (1988) Assessing the benefits of cooperation in honeybee foraging: search costs, forage quality, and competitive ability. Behav. Ecol. Sociobiol. 22, 229-237

Tautz, J. (1996) Honeybee waggle dance: recruitment success depends on the dance floor. J. Exp. Biol. 199, 1375-1381

Tautz, J., Hubland, A. (2008) The buzz about bees: biology of a superorganism. Springer, Berlin

Tautz, J., Lindauer, M. (1997) Honeybees establish specific sites on the comb for their waggle dances. J. Comp. Physiol. A 180, 537-539

Towne, W.F., Gould, J.L. (1988) The spatial precision of the honey bee's dance communication. J. Insect Behav. 1, 129-155

Visscher, P.K., Seeley, T.D. (1982) Foraging strategy of honeybee colonies in a temperate deciduous forest. Ecology 63, 1790-1801

Weidenmuller, A., Seeley, T.D. (1999) Imprecision in waggle dances of the honeybee (Apis mellifera) for nearby food sources: error or adaptation? Behav. Ecol. Sociobiol. 46, 190-199

Zhao, Q., Tao, H. (2005) Object tracking using color correlogram. Proceedings of the 2nd Joint IEEE International Workshop on Visual Surveillance and Performance Evaluation of Tracking and Surveillance (VS-PETS), pp. 263-270 